\title{
Villes d'Europe : à chaque pays sa définition
}

Monsieur Jean-Pierre Le Gléau, Madame Denise Pumain, Madame Thérèse SaintJulien

\section{Citer ce document / Cite this document :}

Le Gléau Jean-Pierre, Pumain Denise, Saint-Julien Thérèse. Villes d'Europe : à chaque pays sa définition. In: Economie et statistique, $n^{\circ}$ 294-295, Mai 1996. Regard socioéconomique sur la structuration de la ville. pp. 9-23;

doi : https://doi.org/10.3406/estat.1996.6079

https://www.persee.fr/doc/estat_0336-1454_1996_num_294_1_6079

Fichier pdf généré le 15/05/2018 


\section{Resumen}

Ciudades de Europa : a cada pafs su definiciôn

Las ciudades europeas, marcadas pour una historia comûn, se desarrollaron en la mayon'a de los casos segûn un modelo de crecimiento concéntrico partiendo de un centra antiguo. Las definiciones que se retienen para las aglomeraciones varfan con creces de un pais a otro.

Existen dos enfoques complementarios para taies definiciones : uno abarca las relaciones econômicas entre el nûcleo urbano y su entorno y define unas "esteras de influencias" de las ciudades. Otro se refiere a la definiciôn del medio urbano en términos de densidad de la construcciôn : este délimita unas unidades urbanas que pueden llegar a formar conurbaciones. Pero, en esta perspectiva, las comparaciones intemacionales son dificiles. En la mayon'a de los pafses (y en Francia en especial), se definen primero unas âreas de la construcciôn continua en torno a de un centra. La unidad urbana es el resultado luego de un ajuste de estas âreas sobre los limites de las circunscripciones administrativas o estadfsticas que las cubren.

El tamarïo de las circunscripciones de base (en general municipios o eslabôn inframunicipal) varîa sin embargo con creces de un pafs a otro (de un a cien entre Francia y Suecia, situadas ambas a cada extremidad de la escala).

Pero en otros pafses, el calificativo de ciudad atribuido a un municipio se debe al estatuto jurfdico o administrative (Alemania), o al salto de un umbral de poblaciôn (Espana, Italia, Portugal) : no se toma en cuenta por tanto la trama de la construcciôn.

En resumen, cinco pafses no tienen definiciôn para la nociôn de unidad urbana ; en los otros, las disparidades del eslabôn de base o de los umbrales retenidos limitan el alcance de las comparaciones intemacionales. Asî es como la definiciôn francesa puede resultar muy extensiva en comparaciôn con la que se retiene en los demâs pafses, teniendo en cuenta la inferioridad del umbral de poblaciôn que aquélla retiene (2000 habitantes).

\footnotetext{
Abstract

The Towns of Europe: To Each Country its Definition

Europe's towns bear the stamp of their shared history. Most of them developed around an ancient centre in line with a concentric growth model. However, the definitions chosen for these towns vary considerably from one country to the next.

There are two complementary approaches to such definitions. The first focuses on the economic relations between the urban core and its environment and defines the towns' "spheres of influence". The second defines the town in terms of building density: it determines urban areas likely to join up into conurbations. Yet even when using this second interpretation, it is difficult to make international comparisons. In most of the countries (especially in France), the uninterrupted built-up zones around a centre are defined first. The urban area then results from adjusting these zones to the administrative or statistical districts that cover them.

The size of the basic districts (generally local administrative areas or interconnected inframunicipal areas) varies considerably from one country to the next (from one for France and to one hundred for Sweden at the opposite end of the scale).

Yet in other countries, a municipality is called a "town" when it has a certain legal or administrative status (Germany) or when it tops a certain population threshold (Spain, Italy and Portugal). Here, the building fabric is not even taken into consideration.

A total of five countries have no definition of the notion of an urban area. In the other countries, disparities between basic interconnections and chosen thresholds limit the scope of international comparisons. This is why the French definition, with its low population threshold $(2,000$ inhabitants $)$ can appear to be extremely extensive compared with that used in the other countries.
}

\section{Résumé}

Villes d'Europe : à chaque pays sa définition

Les villes européennes, marquées par une histoire commune, se sont développées pour la plupart selon un modèle de croissance concentrique à partir d'un centre ancien. Les définitions retenues pour les agglomérations sont cependant différentes d'un pays à l'autre. Il existe deux approches complémentaires pour de telles définitions : l'une s'attache aux relations économiques entre le noyau 
urbain et son environnement et définit des «sphères d'influence » des villes. L'autre concerne la définition du milieu urbain en termes de densité du bâti : elle délimite des unités urbaines elles-mêmes susceptibles de se regrouper en conurbations.

Même dans cette seconde perspective, les comparaisons internationales s'avèrent délicates. Dans la majorité des pays (et en France en particulier), on définit en premier lieu des aires de bâti continu autour d'un centre. L'unité urbaine résulte ensuite d'un ajustement de ces aires sur les limites des circonscriptions administratives ou

ques qui les recouvrent. Toutefois, la taille des circonscriptions de base (en général communes ou maillage inframu- nicipal) varie considérablement entre les pays (de un à cent entre la France et la Suède). Dans d'autres pays, le qualificatif de ville attribué à une municipalité résulte d'un statut juridique ou administratif (Allemagne), ou du franchissement d'un seuil de population (Espagne, Italie, Por- tugal) : le tissu du bâti n'est pas alors pris en considération.

Au total, cinq pays ne disposent pas de définition pour la notion d'unité urbaine ; dans les autres, les disparités du maillage de base ou des seuils retenus limitent la portée des comparaisons internationales. C'est ainsi que la définition française peut apparaître très extensive par rapport à celle retenue dans les autres pays, compte tenu de la faiblesse du seuil de population qu'elle retient ( 2000 habitants).

\section{Zusammenfassung}

Stâdte in Europa: jedem Land seine eigene Definition

Die europâischen Stâdte, die durch eine gemeinsame Geschichte geprâgt sind, haben sich in den meisten Fallen nach einem konzentrischen Wachstumsmodell auf der Grundlage eines alten Zentrums entwickelt. Die Definitionen, die fur dièse Agglomerationen gewâhlt worden sind, unterscheiden sich jedoch erheblich zwischen den einzelnen Làndern.

Fur solche Begriffsbestimmungen gibt es zwei komple- mentâre Ansâtze: der eine stùtzt sich auf die wirtschaft- lichen Beziehungen zwischen dem Stadtkern und seiner Umgebung und bestimmt die "EinfluBsphàren" der Stâdte. Beim zweiten Ansatz werden die stâdtischen Gebiete anhand der Bebauungsdichte definiert, wobei die Stadteinheiten, die Conurbationen bilden kônnen, voneinander abgegrenzt werden. Doch auch bei dieser zweiten Vorgehensweise erweisen sich internationale Vergleiche als recht schwierig. In den meisten Lândern (insbesondere in Frankreich) werden zunâchst die um ein Zentrum liegenden geschlossenen Bebauungsgebiete definiert. Die Stadteinheit ergibt sich somit aus einer Anpassung dieser Gebiete an die Grenzen der adminis- trativen oder statistischen Bezirke, die sie abdecken.

Die GrôBe der Grundbezirke (im allgemeinen Gemeinden oder innerstâdtisches Maschennetz) sind allerdings von Land zu Land sehr unterschiedlich (beispielsweise in einem Verhâltnis von eins zu hundert zwischen Frankreich und Schweden, die die beiden Endpunkte auf der Skala darstellen).

In anderen Lândern ist fur die Bezeichnung "Stadf jedoch der rechtliche oder administrative Status (in Deutschland) oder die Ùberschreitung einer Bevôlkerungsmindestzahl (in Italien, Portugal, Spanien) entscheidend, weshalb die Bebauungsdichte unberùcksichtigt bleibt.

In fûnf Lândern gibt es keine genaue Definition des Begriffes "Stadteinheit". In den anderen werden die internationalen Vergleiche durch die unterschiedliche Grundmaschenstruktur oder durch die gewâhlten Bevôlkerungsmindestzahlen eingeschrânkt. Somit mag die franzôsische Begriffsbestimmung im Vergleich zu denen der anderen Lânder aufgrund der niedrigen Bevôlkerungsmindestzahl (2.000 Einwohner) als recht weitgefaBt erscheinen. 


\section{Villes d'Europe : à chaque pays sa définition}

Jean-Pierre

Le Gléau, Denise Pumain et Thérèse Saint-Julien *

* Jean-Pierre Le Gléau est chef de la division Méthodes comparées de I'Insee. Denise Pumain et Therèse Saint-Julien sont professeurs à l'Université Paris I et membres de l'équipe PARIS (URA 1243 du CNRS). Denise Pumain travaille aussi à l'Institut national d'études démographiques (Ined).

Cet article s'inspire largement d'un rapport établi à la demande d'Eurostat (" Le concept statistique de la ville en Europe "), qu'il actualise pour tenir compte notamment de l'élargissement de l'Union européenne. Denise Pumain et Thérèse Saint-Julien étaient les rédactrices de ce rapport.

Les noms et dates entre parenthèses renvoient à la bibliographie en fin d'article.

Les villes européennes, marquées par une histoire commune, se sont développées pour la plupart selon un modèle de croissance concentrique à partir d'un centre ancien. Les définitions retenues pour les agglomérations sont cependant différentes d'un pays à l'autre. Il existe deux approches complémentaires pour de telles définitions : l'une s'attache aux relations économiques entre le noyau urbain et son environnement et définit des « sphères d'influence » des villes. L'autre concerne la définition du milieu urbain en termes de densité du bâti : elle délimite des unités urbaines elles-mêmes susceptibles de se regrouper en conurbations.

Même dans cette seconde perspective, les comparaisons internationales s'avèrent délicates. Dans la majorité des pays (et en France en particulier), on définit en premier lieu des aires de bâti continu autour d'un centre. L'unité urbaine résulte ensuite d'un ajustement de ces aires sur les limites des circonscriptions administratives ou statistiques qui les recouvrent. Toutefois, la taille des circonscriptions de base (en général communes ou maillage inframunicipal) varie considérablement entre les pays (de un à cent entre la France et la Suède). Dans d'autres pays, le qualificatif de ville attribué à une municipalité résulte d'un statut juridique ou administratif (Allemagne), ou du franchissement d'un seuil de population (Espagne, Italie, Portugal) : le tissu du bâti n'est pas alors pris en considération.

Au total, cinq pays ne disposent pas de définition pour la notion d'unité urbaine ; dans les autres, les disparités du maillage de base ou des seuils retenus limitent la portée des comparaisons internationales. C'est ainsi que la définition française peut apparaître très extensive par rapport à celle retenue dans les autres pays, compte tenu de la faiblesse du seuil de population qu'elle retient ( 2000 habitants).

$\mathrm{P}$ aris, Lyon, Marseille, Lille sont, dans l'ordre, les quatre plus grandes agglomérations françaises, celles qui dépassent ou approchent le million d'habitants. Cependant pour la population (cf. tableau 1), la commune de Marseille devance nettement celle de Lyon, et Lille (172000 habitants) n'est en fait que la quatorzième commune de France, derrière Saint-Étienne, Le Havre et Reims... Cela tient au fait que les agglomérations débordent largement le cadre de la commune-centre ; la prise en compte des banlieues modifie sensiblement le classement effectué sur ces dernières : parfois, en l'absence de banlieue, la population de la commune-centre est la même que celle de l'agglomération (Ajaccio, Cholet); d'autres fois, elle n'en représente même pas le dixième (Béthune, 25000 habitants, centre d'une 
agglomération de 262000 habitants). Encore ne tient-on compte que de la partie française des agglomérations ; si l'on retenait leurs prolongements à l'étranger, il est probable, selon la définition retenue, que l'unité urbaine de Lille passerait en deuxième position, devant Lyon et Marseille.

Depuis 1954, l'Insee détermine à chaque recensement le périmètre des unités urbaines, en tenant compte de critères fixes dans le temps, faisant référence à la continuité physique du bâti (cf. encadré 1) ; grâce à la stabilité de cette définition, il est possible d'en mesurer l'extension géographique. Dans la variation de la population d'un recensement à l'autre, on peut ainsi identifier la part qui revient à l'évolution de l'agglomération dans des limites constantes et celle qui est due à son extension territoriale. Compte tenu des définitions retenues, il est très rare qu'au fil des ans une commune « sorte » du périmètre d'une agglomération. Leur élargissement territorial peut se faire par le rattachement d'une commune rurale proche du périmètre de l'agglomération, ou par l' " absorption » en bloc d'une agglomération de taille moins importante et avec laquelle il n'y a désormais plus de solution de continuité (cas de Marseille Aix-en-Provence en 1990).

Malheureusement, s'il y a permanence des définitions et des concepts dans le temps, la comparabilité dans l'espace est beaucoup moins assurée, même en restant dans le cadre européen ; on ne sait pas quelle est l'extension hors de France des agglomérations frontalières, que la ville principale soit située en France (Lille, Strasbourg) ou à l'étranger (Genève). On ne peut pas non plus comparer, avec l'assurance de se référer à des définitions homogènes, Paris à Londres, Lyon à Milan, ni Toulouse à Saragosse.

\section{Plusieurs concepts derrière le mot « ville »}

Chaque pays a sa propre définition de la « ville »; cette situation s'explique par des considérations géographiques, historiques et administratives. L'approche statistique du fait urbain s'appuie nécessairement sur des représentations de ce qu'est une ville - que cette notion soit définie d'après des savoirs populaires, juridiques ou académiques - et sur des mesures de l'objet urbain ainsi défini. Les villes sont cependant des objets trop riches et trop divers pour qu'une seule définition, une conception unique, puisse en rendre compte. Selon que l'intérêt est porté au cadre bâti, à l' architecture, à l'habitat, aux activités et aux fonctions urbaines, au statut politique ou encore aux modes de vie des habitants, les définitions changent et les mesures aussi.

Dans certains pays, c'est le statut juridique qui identifie la ville. Cette dernière coüncide alors avec ses limites administratives. Pour d'autres, l'urbanité est d'abord une façon d'habiter particulière, et c'est alors la continuité des constructions, la qualité de la desserte par les réseaux de voirie, de transport et d'équipements urbanistiques divers qui confèrent la qualité urbaine à un espace donné. D'autres pri-

\section{Tableau 1}

\section{Les plus grandes « villes » de France (1990)}

En milliers d'habitants

\begin{tabular}{|c|c|c|c|c|c|}
\hline Rang & Commune & Population & Rang & Unité urbaine & Population \\
\hline 1 & Paris & 2152 & 1 & Paris & 9319 \\
\hline 2 & Marseille & 801 & 2 & Lyon & 1262 \\
\hline 3 & Lyon & 415 & 3 & Marseille - Aix-en Provence & 1231 \\
\hline 4 & Toulouse & 359 & 4 & Lille (1) & 959 \\
\hline 5 & Nice & 342 & 5 & Bordeaux & 696 \\
\hline 6 & Strasbourg & 252 & 6 & Toulouse & 650 \\
\hline 7 & Nantes & 245 & 7 & Nice & 517 \\
\hline 8 & Bordeaux & 210 & 8 & Nantes & 496 \\
\hline 9 & Montpellier & 208 & 9 & Toulon & 438 \\
\hline 10 & Rennes & 199 & 10 & Grenoble & 405 \\
\hline
\end{tabular}

Source : recensement de la population de 1990. 
vilégient la fonction économique et considèrent comme urbains tous les établissements humains qui ne remplissent pas principalement des fonctions de production agricole, d'autres associent aux villes tous les habitants qui y passent une partie de leur journée d'activité, tous ceux dont l'essentiel des relations de travail s'effectue en ville, même si leur résidence est ailleurs.

À la complexité des points de vue sur la ville s'ajoute la difficulté d'en délimiter les con- tours, qui sont devenus de plus en plus flous dans l'espace et dans le temps. Les murs d'enceinte qui séparaient deux mondes aux lois distinctes ont partout disparu. L'élévation des niveaux de vie et le développement des transports ont affranchi les citadins de la nécessité d'habiter un périmètre bien circonscrit, bâti en continuité. Des activités et des résidences se diluent dans des zones naguère franchement rurales. Statistiquement, ces nouvelles formes d'urbanisation sont de plus en plus difficiles à saisir.

\section{Encadré 1}

\section{LA DÉLIMITATION DES UNITÉS URBAINES EN FRANCE}

L'unité de base du découpage administratif de la France est la commune et c'est en termes de communes entières que sont définies les zones géographiques appelées unités urbaines : les frontières de ces zones coïncident dans tous les cas avec des limites communales. En revanche, les limites des autres circonscriptions administratives (cantons, arrondissements, départements) ne sont pas prises en considération lors de leur délimitation ; une même unité urbaine peut s'étendre sur deux ou plusieurs départements par exemple et même déborder des frontières nationales. Dans ce dernier cas cependant, l'extension de l'unité urbaine à l'étranger est seulement signalée, son périmètre n'étant pas défini par l'Insee.

À l'occasion du recensement de 1990, les unités urbaines ont été délimitées en plusieurs étapes.

Préalablement au recensement, sur la base des résultats du recensement précédent, des cartes et des photos aériennes les plus récentes et, le cas échéant, à la suite de déplacement sur le terrain, l'on a tout d'abord identifié sur le territoire les zones bâties susceptibles d'atteindre 2000 habitants au recensement de 1990.

Une zone bâtie est constituée par des constructions avoisinantes formant un ensemble tel qu'aucune ne soit séparée de la plus proche de plus de 200 mètres. Les terrains utilisés à des fins publiques tels que jardins publics, aérodromes, routes, cimetières, constructions publiques, ceux utilisés à des fins industrielles ou commerciales tels qu'usines, magasins, édifices commerciaux, voies ferrées, parcs de stationnement..., ainsi que les cours d'eau traversés par des ponts ne sont pas pris en compte lors de la détermination de la distance séparant les habitations.

L'on s'est tout d'abord intéressé aux zones bâties qui s'étendaient sur deux ou plusieurs communes. Parmi les communes concernées, on a éliminé celles dont la population appartenant à la zone bátie représentait moins de la moitié de la population de la commune. Si les communes non éliminées étaient au moins au nombre de deux et si la partie de leur population qui résidait dans la zone atteignait au total au moins 2000 habitants, ces communes constituaient une agglomération multicommunale.

À l'issue du recensement, les communes qui n'appartiennent pas à une agglomération multicommunale délimitée préalablement au recensement ont été classées comme urbaines au sens de l'Insee lorsque le nombre d'habitants de la plus grande zone bâtie (au sens précédemment défini) de la commune atteignait au moins 2000 . Ces communes urbaines sont également appelées villes isolées.

Finalement, on a appelé unités urbaines aussi bien les agglomérations multicommunales que les villes isolées. Au sens de l'Insee, toute commune appartenant à une unité urbaine est réputée " urbaine ", toutes les autres communes étant classées " rurales".

Pour chacune des agglomérations multicommunales, on a défini un " centre ". II s'agit d'un ensemble composé d'une ou plusieurs communes entières déterminées comme suit : si une commune représente plus de $50 \%$ de la population de l'unité urbaine, elle est seule ville-centre. Dans le cas contraire, toutes les communes qui ont une population supérieure à la moitié de la population de la commune la plus importante, ainsi que cette dernière, sont villes-centres. Les communes urbaines qui ne sont pas villes-centres constituent la banlieue de l'unité urbaine.

Les agglomérations multicommunales sont au nombre de 896 (dont 892 en métropole et 4 dans les DOM) ; elles comprennent 4314 communes (4 301 en métropole, 13 dans les DOM) classées comme urbaines au sens de l'Insee. Par ailleurs, les villes isolées telles qu'elles résultent du recensement de 1990 sont au nombre de 1065 (dont 999 en métropole et 66 dans les DOM). Au total, il y a donc 1961 unités urbaines ( 1891 en métropole, 70 dans les DOM) comprenant 5379 communes urbaines (5 300 en métropole, 79 dans les DOM). Les communes rurales sont au nombre de 31285 (dont 31251 en métropole et 34 dans les DOM). 
Si ces phénomènes se retrouvent d'un pays à l'autre, ils ont pris et prennent encore des traductions différentes dans l'aspect et l'organisation spatiale des zones urbanisées. Des facteurs importants pour l'urbanisation comme la densité du peuplement, les normes admises et les règlements d'urbanisme, les formes d'appropriation du sol et du bâti, la plus ou moins grande force d'intervention des organismes publics de planification et de régulation, varient beaucoup selon les pays, voire selon les régions d'Europe. Les diverses phases de l'histoire de l'urbanisation, bien que communes à l'ensemble des pays européens et de plus en plus simultanées, ont ainsi pu produire des tissus urbains qu'il est difficile de caractériser par des mesures semblables, que ce soit en termes de densités de population, de logements ou d'activités, de continuité de constructions, ou d'organisation des quartiers.

\section{La spécificité des villes européennes}

L'observation des villes et l'examen des définitions et des statistiques existantes montrent une certaine spécificité du fait urbain européen, par exemple quand on le compare aux villes d'Amérique du Nord, continent d'urbanisation récente. En dépit de la variété des tissus urbains, des inégalités des maillages administratifs, des différences de densité de population et de densité du bâti d'un pays à l'autre, en dépit de l'existence de plusieurs générations de villes qui se sont développées à des moments différents de l'histoire et des techniques, les villes d'Europe se caractérisent par leur développement concentrique et par la grande lisibilité $d u$ paysage urbain, qui tient à une organisation particulière. La formation urbaine caractéristique, celle qui est la plus fréquente et qui se trouve représentée dans tous les pays, comprend un noyau central plus ancien et plus dense, une zone développée plus ou moins concentriquement en continuité de bâti avec une forte compacité, et des extensions périphériques plus lâches.

C'est encore dans la zone centrale d'urbanisation dense que reste localisé l'essentiel des résidents, mais surtout des activités économiques, des emplois et des fonctions urbaines. Les tendances récentes de l'urbanisation ont fait évoluer la forme des villes dans deux directions, apparemment contradictoires.

Le premier type de transformations tendrait à rapprocher les villes européennes du modèle américain : la déconcentration des populations urbaines, les implantations en périphérie des installations industrielles consommatrices d'espace, de centres commerciaux et administratifs près des voies de communication, et d'activités techniques avancées près des aéroports, ont compliqué le schéma d'organisation des villes, auxquelles sont désormais associés des pôles secondaires de résidence et d'activités qui peuvent être isolés du noyau urbain principal par des espaces ruraux, apportant des discontinuités dans le tissu urbain.

Dans le même temps, une tendance inverse faite de rénovation du bâti, de réinvestissement social résidentiel, d'un renforcement de la pression foncière et de la concentration des activités les plus prestigieuses dans les vieux centres s'est dessinée dans presque toutes les villes du continent, contribuant à revaloriser et à confirmer la spécificité du modèle des villes européennes.

\section{Deux approches complémentaires de l'urbanisation}

Pour décrire cette situation, deux points de vue peuvent être adoptés : ou bien on essaye de définir des espaces correspondant à des degrés différents d'urbanisation (la façon la plus brutale étant de ne distinguer que deux catégories : « urbain » et « rural »); ou bien on délimite autour de chaque noyau urbain une sphère d'influence, rattachant toute commune au noyau urbain avec lequel elle a le plus de relations. Ces deux points de vue ne sont pas contradictoires, mais complémentaires. Une représentation géographique du premier conduit à une carte parsemée de taches - situant les pôles les plus manifestement urbains - éventuellement entourées d'une ou plusieurs couronnes correspondant à des espaces présentant des caractéristiques urbaines moins affirmées. La toile de fond de telles cartes est constituée d'un espace résiduel, généralement appelé espace rural, ayant lui-même sa propre structuration, répondant cependant à une logique différente de celle de l'urbain, et donc mesurée à l'aide de critères différents. Le deuxième point de vue conduit à un maillage plus ou moins fin de l'espace total, ne laissant pas, contrairement au précédent, d'espace interstitiel. Ce qui caractérise les communes d'une même cellule de ce zonage, ce n'est pas un même $d e$ gré d'urbanisation, mais une direction commune vers laquelle s'orientent certaines relations - bien souvent les déplacements domicile-travail - ; associant villes et campagnes, 
les zones ainsi constituées concrétisent les solidarités pouvant exister entre communes ayant des degrés d'urbanisation différents.

Dans la suite de cet article, on s'attachera à présenter les options retenues par les divers pays de l'Union européenne pour effectuer la dichotomie urbain/rural et déterminer les unités urbaines. La nécessité d'une telle distinction apparaît chaque fois que l'on se pose des questions relatives au rapport à l'espace des populations, au dimensionnement des réseaux de transports et de communications, à la localisation des équipements de services, à la probabilité de diffusion d'innovations...

C'est pourtant l'une des caractéristiques les plus difficiles à justifier, puisque dans aucun pays il n'existe plus de limite nette entre la campagne et la ville : du point de vue morphologique comme sur le plan statistique, il n'y a pas de césure, non seulement dans la distribution de la dimension des agrégats de population (on a au contraire un « continuum rural-urbain»), mais encore pour toutes sortes d'indicateurs socioéconomiques. En outre, dans bien des pays, et surtout depuis une quarantaine d'années, les processus successifs d'extension des zones urbanisées, de suburbanisation (zones urbaines de banlieue), de péri-urbanisation (zones rurales investies par des résidents travaillant en ville) et de rurbanisation (diffusion de population urbaine dans des zones rurales plus éloignées) ont considérablement emmêlé les frontières entre villes et campagnes.

\section{Unités urbaines et conurbations}

Les unités urbaines que l'on peut mettre en évidence comme résultat de cette distinction se réfèrent d'abord à des entités morphologiques. Elles sont définies le plus souvent d'après la continuité du bâti, d'après la cohérence du tissu urbain. Ce sont ces entités qui sont donc pertinentes pour aborder toutes les questions de gestion urbanistique et d'équipements du type voirie, éclairage public, assainissement... notamment chaque fois qu'est requise la coopération entre des collectivités territoriales différentes. Mais, en Europe, c'est également dans l'agglomération que se localise la plus grande partie des activités, des fonctions et des emplois urbains, si bien qu'elle constitue aussi un cadre pertinent pour les mesures et les comparaisons, nationales ou internationales, du poids et de l'importance des villes d'un point de vue économique (Cattan et alii, 1994).
Dans certaines régions, des agglomérations constituées autour de noyaux urbains distincts se sont rejointes et sont désormais bâties en continuité. Ces conurbations ou agglomérations multipolaires sont à prendre en considération lorsque sont posées les questions de l'aménagement concerté de vastes espaces urbanisés ; ce sont aussi des lieux privilégiés pour analyser la formation de réseaux des spécialisations des villes. Le cas typique en Europe est celui de la Ruhr où une longue conurbation s'étire du nord au sud, de Duisburg à Bonn et Cologne, et d'est en ouest, de Dortmund à Düsseldorf et Moenchengladbach, sans qu'un pôle dominant apparaisse clairement. Cette configuration est peu fréquente en France, où elle ne se rencontre que dans quelques régions (Nord, Côte d'Azur...).

Compte tenu du rôle joué par la trame communale dans la définition des populations et des unités urbaines, il est nécessaire de rappeler quelques caractéristiques des découpages territoriaux qui forment la base du système statistique.

\section{De grandes communes dans le Nord de l'Europe}

Dans la plupart des pays, les municipalités forment le premier échelon auquel correspond une division administrative du territoire national. Par leur surface et par la population qu'elles concentrent, ces subdivisions ont des dimensions moyennes qui varient beaucoup d'un pays à l'autre (cf. tableau 2).

Cinq pays se détachent nettement par la grande dimension de ces mailles de base, et par l'importance de la population qu'elles contrôlent. La Suède, où les kommuner ont en moyenne $1437 \mathrm{~km}^{2}$ pour plus de 30000 habitants, la Finlande, dont les kunnat ont $743 \mathrm{~km}^{2}$ et 11100 habitants et l'Irlande avec des districts dont la superficie moyenne est de $766 \mathrm{~km}^{2}$ et la population moyenne de 39400 habitants, arrivent en tête. Viennent ensuite le Royaume-Uni, dont les districts atteignent en moyenne $500 \mathrm{~km}^{2}$ et 120000 habitants, et le Portugal, avec des concelhos dont la surface moyenne est de $302 \mathrm{~km}^{2}$ et la population de 32300 habitants.

Très en deçà des précédentes, les surfaces moyennes des communes belges $\left(52 \mathrm{~km}^{2}\right)$, des gemeenten néerlandaises $\left(61 \mathrm{~km}^{2}\right)$ et des municipios espagnoles $\left(62 \mathrm{~km}^{2}\right)$ sont très proches. À ces maillages administratifs élémentaires 
correspondent, en moyenne, des concentrations de population voisines en Belgique et aux PaysBas (respectivement 17100 et 22600 habitants), mais beaucoup plus modestes pour l'Espagne (4 800 habitants).

Les comuni italiennes $\left(37 \mathrm{~km}^{2}\right)$, les Gemeinden autrichiennes $\left(36 \mathrm{~km}^{2}\right)$ ou allemandes $\left(24 \mathrm{~km}^{2}\right)$, les demoi grecques et les communes luxembourgeoises $\left(22 \mathrm{~km}^{2}\right)$ sont des mailles élémentaires de superficie moyenne plus réduite. Les populations moyennes qu'elles concentrent les différencient davantage : plus élevées en République fédérale d'Allemagne (5 500 habitants) et en Italie ( 7000 habitants), plus faibles en Autriche (3 400 habitants), au Luxembourg (3 300 habitants) et en Grèce (1 700 habitants). Les freguesias portugaises (subdivision des concelhos déjà nommés) et les församling (paroisses) suédoises se rapprochent de ces unités : elles concentrent en moyenne respectivement 2300 et 3500 habitants.

Les communes françaises occupent le dernier rang, avec une surface moyenne d'à peine $17 \mathrm{~km}^{2}$ et une population de seulement 1600 habitants (et même $15 \mathrm{~km}^{2}$ et 1500 habitants pour la seule métropole). C'est là une situation très singulière parmi les pays de taille comparable.

Ces dimensions moyennes ne sont cependant pas entièrement représentatives d'une plus ou moins grande finesse de maillage qui serait propre à chaque pays : on doit noter qu'à l'intérieur de chacun des pays la variance intermunicipale des tailles est toujours très importante, en surface comme en population (cf. encadré 2). Ainsi, en France, la superficie moyenne des communes varie de $5 \mathrm{~km}^{2}$ dans les Hauts-deSeine à $43 \mathrm{~km}^{2}$ dans les Bouches-du-Rhône, et la population moyenne de moins de 400 habitants dans le Gers, la Meuse ou la Lozère à plus de 8000 en Île-de-France.

Ces disparités constituent un premier obstacle à la comparaison internationale des villes. En effet, même si les définitions retenues pour les unités urbaines étaient formellement les mêmes, on ne pourrait pas accorder le même sens à un indicateur calculé au RoyaumeUni pour un district de plus de 100000 habitants et en France pour une commune d'à peine plus de 1000 habitants; par exemple le critère

Tableau 2

Les unités locales

\begin{tabular}{|c|c|c|c|c|}
\hline Pays & Nom des unités locales & Nombre & $\begin{array}{l}\text { Superficie moyenne } \\
\left(\mathrm{km}^{2}\right)\end{array}$ & $\begin{array}{l}\text { Population moyenne } \\
\text { (1000 habitants) }\end{array}$ \\
\hline Allemagne & Gemeinden & 14742 & 24 & 5,5 \\
\hline Autriche & Gemeinden & 2353 & 36 & 3,4 \\
\hline Belgique & Communes & 589 & 52 & 17,1 \\
\hline Danemark & Kommuner & 276 & 156 & 18,7 \\
\hline Espagne & Municipios & 8077 & 62 & 4,8 \\
\hline Finlande & Kunnat & 455 & 743 & 11,1 \\
\hline France & Communes & $3666 i$ & 17 & 1,6 \\
\hline Grèce & Demoi / koinotites & 5921 & 22 & 1,7 \\
\hline \multirow[t]{2}{*}{ Irlande } & Districts & 90 & 766 & 39,4 \\
\hline & DEDs / wards & 3445 & 20 & 1,0 \\
\hline Italie & Comuni & 8100 & 37 & 7,0 \\
\hline Luxembourg & Communes & 118 & 22 & 3,3 \\
\hline Pays-Bas & Gemeenten & 672 & 61 & 22,6 \\
\hline \multirow[t]{2}{*}{ Portugal } & Concelhos - municipos & 305 & 302 & 32,3 \\
\hline & Freguesias & 4208 & 22 & 2,3 \\
\hline \multirow[t]{2}{*}{ Royaume-Uni } & Districts & 485 & 499 & 119,6 \\
\hline & Wards & 11095 & 22 & 5,2 \\
\hline \multirow[t]{2}{*}{ Suède } & Kommuner & 288 & 1437 & 30,3 \\
\hline & Församling & 2545 & 161 & 3,5 \\
\hline
\end{tabular}

Sources : Eurostat et sources nationales. 
« travailler et résider dans la même commune » ou un seuil de taille ( « commune de plus de... $x$ habitants ») n'ont pas le même sens dans deux découpages communaux aussi différents.

Sur ces trames communales et administratives variées, ce sont d'abord le législateur puis le statisticien qui ont distingué les unités urbaines et les zones rurales. Les principes actuels de définition du caractère urbain relèvent encore parfois d'une décision administrative, mais plus souvent de critères statistiques de taille de la population communale ou d'agglomérations de peuplement repérées sur le terrain. C'est géné-

Encadré 2

MOYENNE OU MÉDIANE ?

Le calcul des moyennes peut être influencé par l'existence de très grosses unités. Ainsi la population moyenne des communes françaises est-elle de 1584 habitants ; mais elle ne serait que de 1525 habitants si l'on excluait la ville de Paris et tomberait même à 1345 si l'on n'effectuait le calcul que sur les 36328 communes de moins de 100000 habitants. La taille moyenne des communes ne correspond pas à la taille de la " commune moyenne " telle qu'on peut l'imaginer spontanément. Celle-ci serait plutôt donnée par la médiane ; c'est la taille qui partage l'ensemble des communes en deux parties ayant le même nombre d'unités : il y a autant de communes plus grosses que la médiane qu'il y a de communes plus petites qu'elle.

Appliqué à la population, le calcul de la médiane donne effectivement des résultats différents de la moyenne (cf. tableau A). Les Städte et Gemeinden sont en moyenne plus peuplées en Allemagne qu'en

Tableau A

Population des unités locales

En milliers d'habitants

\begin{tabular}{|l|c|c|}
\hline & Moyenne & Médiane \\
\hline Suède (kommuner) & 30,3 & 16,0 \\
Pays-Bas (gemeenten) & 22,6 & 11,9 \\
Danemark (kommuner) & 18,7 & 9,8 \\
Belgique (communes) & 17,1 & 10,7 \\
Finlande (kunnat) & 11,1 & 5,0 \\
Italie (comuni) & 7,0 & 2,3 \\
Royaume-Uni (wards) & 5,2 & 3,9 \\
Allemagne (Gemeinden) & 5,5 & 0,9 \\
Espagne (municipios) & 4,8 & 0,6 \\
Autriche (Gemeinden) & 3,4 & 1,6 \\
Luxembourg (communes) & 3,3 & 1,4 \\
Portugal (freguesias) & 2,3 & 1,0 \\
Grèce (demoi) & 1,7 & 0,4 \\
France (communes) & 1,6 & 0,4 \\
Irlande (DEDs/wards) & 1,0 & 0,5 \\
\hline
\end{tabular}

Source : Eurostat.
Autriche ; mais le calcul de la médiane donne un résultat inverse, en raison de l'existence de très grosses villes en Allemagne. Une conclusion analogue peut être tirée de la comparaison des tailles moyennes et médianes des freguesias portugais et des municipios espagnols.

Les distributions des tailles des communes étant toujours très dissymétriques, beaucoup de petites communes et peu de très grandes, la population médiane est toujours inférieure à la population moyenne : d'assez peu au Royaume-Uni et dans les pays du Nord de l'Europe (Allemagne exceptée), de beaucoup dans l'Europe méditerranéenne (Espagne, France, Grèce, Italie) en raison d'un mode de découpage du territoire qui privilégie quelques grosses unités.

La comparaison analogue effectuée sur les superficies moyennes et médianes met en évidence des disparités beaucoup moins fortes (cf. tableau B).

\section{Tableau B}

\section{Superficie des unités locales}

\begin{tabular}{|l|r|c|}
\multicolumn{2}{c}{} & En km \\
\hline & Moyenne & Médiane \\
\hline Suède (kommuner) & 1437 & 676 \\
Finlande (kunnat) & 743 & 364 \\
Danemark (kommuner) & 156 & 143 \\
Espagne (municipios) & 62 & 35 \\
Pays-Bas (gemeenten) & 61 & 35 \\
Belgique (communes) & 52 & 40 \\
Italie (comuni) & 37 & 22 \\
Autriche (Gemeinden) & 36 & 25 \\
Allemagne (Gemeinden) & 24 & 13 \\
Luxembourg (communes) & 22 & 19 \\
Grèce (demoi) & 22 & 15 \\
Portugal (freguesias) & 22 & 11 \\
Royaume-Uni (wards) & 22 & 6 \\
Irlande (DEDs/wards) & 20 & n.d. \\
France (communes) & 17 & 11 \\
\hline
\end{tabular}

Source : Eurostat. 
ralement l'addition des populations résidant dans ces noyaux urbains, ou dans les circonscriptions administratives qui les englobent, qui permet de définir et de mesurer la population urbaine.

L'usage qui est fait de ces classements est également très différent d'un pays à l'autre. Dans certains cas le titre de « ville » donne à certaines municipalités des droits et des devoirs particuliers ; dans d'autres pays, il s'agit d'une simple nomenclature statistique servant de référence pour les études.

\section{La ville définie par un statut administratif particulier}

Plusieurs pays introduisent la notion d'urbain dans l'appellation de leurs unités administratives (cf. tableau 3). Il peut s'agir de la définition d'un statut particulier pour des villes capitales. C'est par exemple le cas du district du Grand Londres au Royaume-Uni, des communes et districts de Copenhague et Frederiksberg au Danemark, des chartes des municipalités de Madrid et de Barcelone en Espagne, ou encore de la définition légale en Irlande de 5 county boroughs, 5 municipal boroughs, 49 urban districts et 31 towns d'après le town improvement act de 1854. En Autriche, 176 communes ont reçu le titre de Stadt (ville), en vertu d'une décision légale prise il y a plus ou moins longtemps (parfois depuis plusieurs siècles); 15 d'entre elles se confondent avec le district, plus petite unité de gestion administrative de l'État. Audelà de cette individualisation, en Angleterre et au Pays de Galles d'une part, en République fédérale d'Allemagne d'autre part, le législateur identifie administrativement des territoires urbains et des territoires qui ne le sont pas.

\section{Tableau 3}

\section{Pays où certaines communes} ont officiellement le titre de « ville »

\begin{tabular}{|c|c|}
\hline $\begin{array}{c}\text { Par décision administrative } \\
\text { ou législative }\end{array}$ & $\begin{array}{l}\text { Par franchissement } \\
\text { d'un seuil de population }\end{array}$ \\
\hline Allemagne & Espagne \\
\hline Autriche & Italie \\
\hline Irlande & Portugal \\
\hline Danemark & \\
\hline Royaume-Uni & \\
\hline $\begin{array}{l}\text { Espagne } \\
\text { (pour Madrid et Barcelone) }\end{array}$ & \\
\hline
\end{tabular}

Toutes ces appellations définies par la loi enregistrent autant l'état des rapports de force politiques entre l'État et les villes à un moment donné que l'importance et l'extension du monde urbain. Elles ne peuvent rendre compte de ce dernier, qui par définition se modifie, alors quc lcs qualités d'une trame administrative reposent sur sa stabilité.

En France, il n'y a pas de définition juridique de la ville; en conséquence, on ne trouve aucune instance de décision politique correspondant aux agglomérations ou unités urbaines.

La plupart des pays ont cherché à définir des normes de l'urbain qui se distinguent des normes administratives.

\section{L'urbain défini par un seuil de population communale}

Pour d'autres pays, la délimitation de la ville est encore tributaire des frontières d'une seule municipalité. Cependant le critère permettant d'attribuer le statut urbain à une municipalité n'est plus d'ordre administratif; il repose sur le nombre d'habitants que concentrent les territoires municipaux. Un seuil de population détermine les municipalités qui sont des villes, et celles qui ne le sont pas.

L'Espagne et l'Italie, par convention, définissent comme villes toutes les communes qui ont au moins 10000 habitants. Leur population est dite urbaine.

Ces définitions sont évidemment fortement tributaires de la finesse du maillage communal.

\section{Définition de l'agglomération morphologique}

Plusieurs pays ont défini la notion d'unité urbaine. Libérée du carcan des limites administratives, la ville est définie à partir d'une aire de bâti continu autour d'un centre. Elle englobe le territoire municipal du centre, et les espaces de banlieue qui se sont progressivement constitués à la périphérie. Une part dominante des activités qui assurent la dynamique économique de la ville restent localisées sur le territoire de ces unités. À l'intérieur de ce périmètre très densément occupé, les économies d'agglomération, qui justifient pour les populations et pour les activités une localisation urbaine, sont maximisées. 
La définition des unités urbaines exige que soit précisée la manière dont on définit une zone de bâti continu. Elle requiert aussi que l'on se donne les outils permettant de suivre la progression géographique de ce bâti. Précisons d'abord ce que recouvrent ces agrégats bâtis élémentaires.

France, Autriche, Belgique, Danemark, Finlande, Suède, Grèce, Irlande et Royaume-Uni définissent des noyaux élémentaires de peuplement. L'idée de base retenue est la suivante : l'urbain est avant tout caractérisé par une agglomération de population, qui se traduit par une masse démographique (le minimum retenu varie selon les pays de 50 à 1000 habitants), et par un seuil de distance qui définit la continuité spatiale et sépare une agglomération d'une autre agglomération. Quand clle cst prćcisćc, cette distance est évaluée à 50 ou à 200 mètres. Elle est mesurée en général en excluant tous les espaces non constructibles, sur des cartes ou des photographies aériennes, ou en recourant à l'observation sur le terrain. Elle ne fait référence qu'à des critères physiques, et ignore totalement les limites administratives avec lesquelles elle ne coïncide qu'exceptionnellement.

Très voisines par leur esprit, les définitions données par chacun des pays sont assez différentes dans le détail (cf. tableau 4).

En France, la zone bâtie est constituée de constructions avoisinantes formant un ensemble tel qu'aucune ne soit séparée de la plus proche de plus de 200 mètres. Deux ensembles de ce type ne sont pas distingués si la distance minimum qui les sépare est de moins de 200 mètres. Les terrains à usage public, ceux utilisés à des fins industrielles et commerciales ainsi que les cours d'eau traversés par des ponts ne sont pas pris en compte dans le calcul de cette distance. Une même commune peut comprendre plusieurs zones bâties. Une définition analogue est retenue en Autriche, mais elle a évolué au cours du temps. À la suite du recensement de 1981 , les statisticiens autrichiens ont commencé par repérer sur des cartes les zones de bâti continu, définies comme ci-dessus. Ils ont ensuite considéré l'ensemble des districts de recensement (fraction du territoire communal) couvrant chaque zone pour ne retenir que ceux dont la moitié au moins de la population vivait dans la zone de bâti continu. La réunion des districts de recensement ainsi sélectionnés formait ce qu'on appelait les «localités » (Siedlungseinheiten). Il n'y avait donc pas coïncidence exacte entre ces dernières et les zones de bâti continu ; l'écart relatif risquait même d'être élevé pour les plus petites unités ; c'est pourquoi les statisticiens autrichiens n'ont défini les localités que si elles abritaient au moins 2000 habitants. En 1991 au contraire, les localités sont définies de façon plus stricte et coïncident exactement avec la zone bâtie ellemême. Dès lors, la restriction sur la taille n'a plus de raison d'être ; toutefois, pour des raisons pratiques, seules les localités de plus de 500 habitants ont reçu une description formelle.

En Belgique, un noyau d'habitat correspond à une partie du territoire contenant un ensemble de maisons avoisinantes, d'édifices publics, de petits établissements industriels ou commerciaux, y compris les voies de communication, les parcs, les terrains de sport, etc. Il est délimité par des terres arables, des bois, des terres incultes, éventuellement parsemés d'habitations dispersées. Cette définition ne fait référence à aucun critère quant à la distance maximale séparant les maisons. Il existe certaines situations où il est malaisé de dire si une zone relève du type d'habitat «aggloméré » ou du type d'habitat « dispersé »; dans ce cas, la délimitation des noyaux d'habitat comporte une certaine part de subjectivité. Seuls ont été pris en compte les noyaux d'habitat ayant une population d'au moins 200 habitants.

Les pays nordiques, qu'ils soient membres de l'Union européenne (Danemark, Finlande, Suède) ou non (Norvège, Islande), ont

\section{Tableau 4}

Définition des zones de bâti continu

\begin{tabular}{|c|c|c|}
\hline & $\begin{array}{l}\text { Distance } \\
\text { maximum } \\
\text { entre les } \\
\text { bâtiments }\end{array}$ & Seuil de population \\
\hline France & $200 \mathrm{~m}$ & $\begin{array}{l}\text { aucun } \\
\text { (50 habitants jusqu'en 1982) }\end{array}$ \\
\hline Autriche & $200 \mathrm{~m}$ & 500 habitant depuis 1991 \\
\hline Belgique & non précisée & 200 habitants \\
\hline $\begin{array}{l}\text { Danemark } \\
\text { Finlande } \\
\text { Suède }\end{array}$ & $\begin{array}{l}200 \mathrm{~m} \\
\text { (modulation } \\
\text { possible) }\end{array}$ & 200 habitants \\
\hline Grèce & $200 \mathrm{~m}$ & aucun \\
\hline Irlande & $200 \mathrm{~m}$ & 50 maisons occupées \\
\hline $\begin{array}{l}\text { Angleterre } \\
\text { Pays de Galles }\end{array}$ & $50 \mathrm{~m}$ & environ 1000 habitants \\
\hline Écosse & $50 \mathrm{~m}$ & 500 habitants \\
\hline
\end{tabular}


harmonisé leur définition de l'agglomération de population, appelée aire ou localité urbaine. Celle-ci doit rassembler au moins 200 habitants, et la continuité de bâti est reconnue dès lors que moins de 200 mètres séparent deux maisons. Les parcs publics, les terrains de sports, les bâtiments à usage industriel ou commercial n'interrompent pas la continuité. Cependant, à l'inverse de ce qui se pratique dans les autres pays, l'application de cette définition ne se fait pas mécaniquement ; un certain degré d'appréciation sur la distance de 200 mètres est par exemple possi- ble lorsque le noyau de l'agglomération n'est pas sensiblement plus compact que l'aire environnante, c'est-à-dire lorsqu'il n' y a pas de limite nette entre population agglomérée et population éparse.

En Grèce, la population agglomérée d'une municipalité ou d'une commune est celle qui réside dans des habitations distantes les unes des autres de moins de 200 mètres. La continuité du bâti n'est pas prise en compte si elle n'est repérable que le long d'une seule voie de communication.

\section{Schéma}

Unité urbaine : à chaque pays sa définition

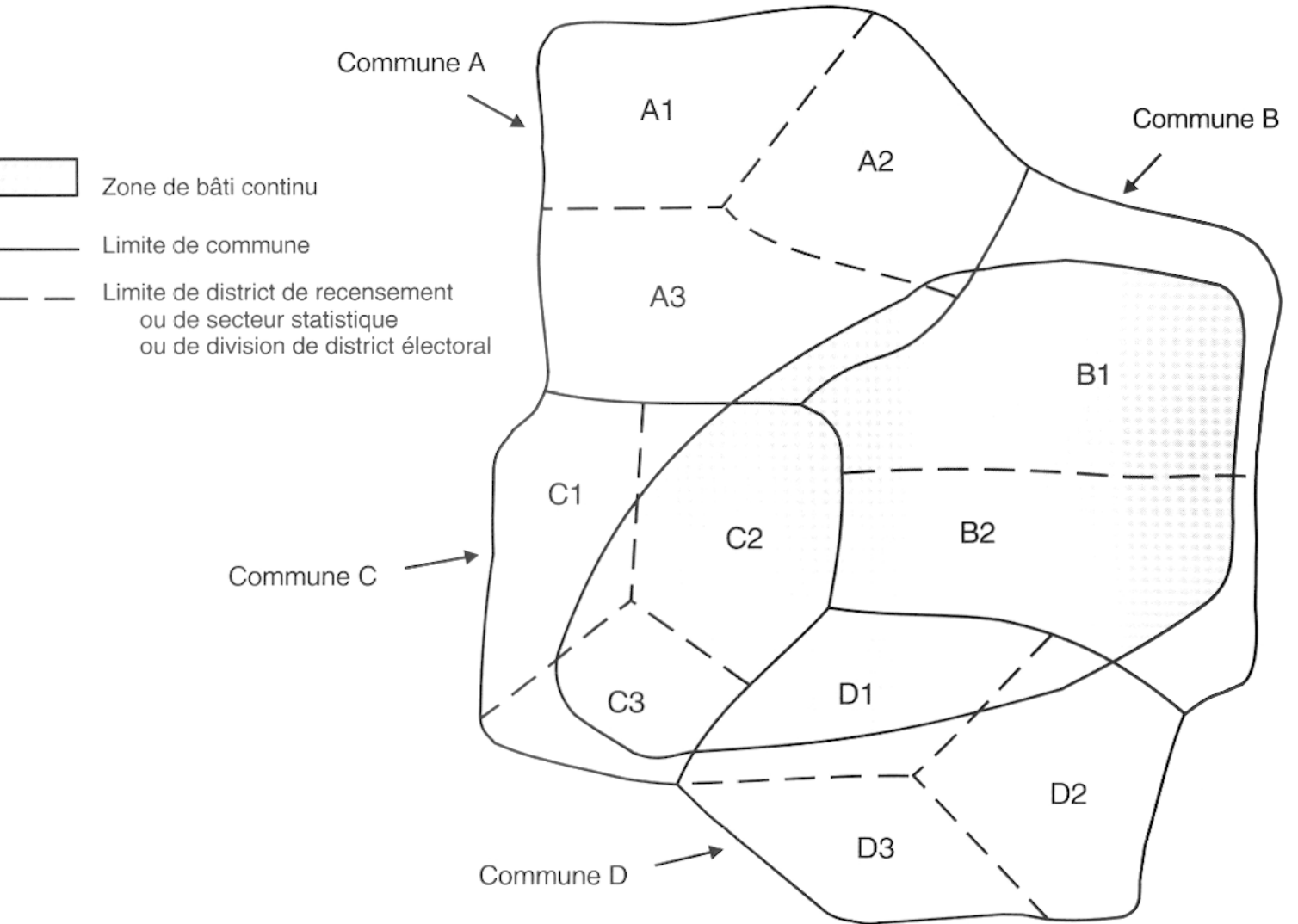

Lecture : sous réserve de conditions sur la taille, les délimitations des unités urbaines sont les suivantes:

- En Belgique, l'agglomération morphologique est composée des ensembles $B_{1}, B_{2}, C_{2}, C_{3}, D_{1}$ (qui normalement coïncident avec la zone hachurée); l'agglomération opérationnelle est composée des communes $B$ et $C$.

- En Suède et au Danemark, l'unité urbaine est identique à la zone hachurée.

- En Irlande, l'aggregated town est composée des ensembles $B_{1}, B_{2}, C_{2}, C_{3}, D_{1}$

- Au Luxembourg l'agglomération urbaine est identique à la zone hachurée; l'aire urbaine est composée des communes $A, B, C, D$.

- Au Royaume-Uni, l'urban area est composée des ensembles $B_{1}, B_{2}, C_{2}, C_{3}, D_{1}$.

- En France, l'unité urbaine est composée des communes $B$ et $C$.

- En Autriche, l'unité urbaine est composée des communes A, B, C et D.

- En Grèce, l'unité urbaine est composée des communes A, B, C et D. 
En Angleterre et au Pays de Galles, la définition première est celle de surface urbanisée (urban land) : il s'agit d'un terrain qui possède au sol des structures permanentes, des axes de circulation qui alignent du bâti continu, au moins sur un des côtés, des bâtiments distants de moins de 50 mètres, des équipements de transport, comme des parkings, ou des aires de desserte d'autoroutes. Quand un terrain urbanisé correspond à une surface d'au moins 20 hectares et regroupe environ 1000 habitants, il est déclaré aire urbaine. Un principe très voisin est appliqué en Écosse, à quelques nuances près cependant. Le seuil de population retenu pour identifier une locality, ou aire de bâti continu n'est que de 500 habitants. Les aires de bâti continu qui, bien que n'atteignant pas 500 habitants lors du recensement de 1971, ont connu entre 1971 et 1981 , une croissance de population importante, sont aussi dites localities. Aucune référence n'est faite à la surface de l'aire de bâti continu. Aucune précision n'est donnée pour l'Irlande du Nord.

En Irlande, l'agglomération de population correspond à 50 maisons occupées et distantes de moins de 200 mètres.

\section{De l'agglomération morphologique à l'unité urbaine}

L'agglomération de population étant définie, la délimitation de l'unité urbaine résulte d'un ajustement sur les limites externes des circonscriptions administratives ou statistiques qui la recouvrent (cf. schéma). On peut distinguer deux groupes de pays. Dans le premier (Danemark, Suède, Irlande, Luxembourg, RoyaumeUni), les territoires des unités urbaines ne sont pas définis par un nombre entier de communes. La délimitation résulte alors d'un ajustement sur un maillage inframunicipal. Dans le second groupe (France, Grèce, Autriche), la municipalité est l'unité élémentaire à partir de laquelle sont constituées les unités urbaines (cf. tableau 5 et encadré 3 ).

Quant à la Belgique, on y trouve deux définitions, qui permettent de classer ce pays dans l'un ou l'autre groupe : la première, qui ferait classer ce pays dans le premier groupe, est celle qui a pour base les secteurs statistiques (ou quartiers); ces derniers représentent une portion du territoire communal aussi homogène que possible : un quartier est donc caractérisé soit par des habitations agglomérées, soit par des habitations dispersées. Un noyau d'habitat est donc obtenu par le regroupement de quartiers dont les habitations sont agglomérées ; il porte le nom d' « agglomération morphologique » lorsqu'il s'étend sur le territoire de plusieurs communes. La deuxième définition, qui apparente la Belgique aux pays du second groupe, correspond à la notion d' " agglomération opérationnelle », obtenue en ajustant le noyau d'habitat aux limites des communes. Une commune est considérée comme faisant partie de l'agglomération opérationnelle si plus de la moitié de sa population réside dans le noyau d'habitat.

Quelques pays définissent les unités urbaines en s'appuyant sur des découpages infracommunaux. Ainsi, en Suède et au Danemark, les zones de bâti continu déterminées, comme nous l'avons vu, indépendamment des limites communales, sont retenues en l'état pour définir les unités urbaines. Rendues publiques par l'autorité administrative, ces délimitations n'ont toutefois pas de valeur légale.

En Irlande, le District Electoral Division est l'unité constitutive de base de l'unité urbaine. À côté des 90 towns définies légalement, le recensement de 1981 dénombre 530 census towns, dont 196 seulement ont plus de 1000 habitants. Des aggregate lown areas doivent comprendre au moins un noyau de population de plus de 1500 habitants.

\section{Tableau 5}

\section{Les définitions des unités urbaines}

\begin{tabular}{|c|c|}
\hline $\begin{array}{l}\text { Danemark } \\
\text { Suède }\end{array}$ & Zone de bâti continu \\
\hline Luxembourg & $\begin{array}{l}\text { Localités définies par des critères } \\
\text { socioéconomiques }\end{array}$ \\
\hline Irlande & Zone de bâti continu ajustée sur les DEDs \\
\hline $\begin{array}{l}\text { Angleterre } \\
\text { Pays de Galles }\end{array}$ & $\begin{array}{l}\text { Zone de bâti continu ajustée sur } \\
\text { l'« enumeration district " }\end{array}$ \\
\hline Écosse & $\begin{array}{l}\text { Zone de bâti continu ajustée sur le local } \\
\text { authority }\end{array}$ \\
\hline Belgique & $\begin{array}{l}\text { Zone de bâti continu ajustée sur : } \\
\text { - les quartiers (agglomération } \\
\text { morphologique); } \\
\text { - les communes (agglomération } \\
\text { opérationnelle) }\end{array}$ \\
\hline France & $\begin{array}{l}\text { Zone de bâti continu de plus de } 2000 \\
\text { habitants ajustée sur les communes }\end{array}$ \\
\hline Grèce & $\begin{array}{l}\text { Zone de bâti continu de plus de } 10000 \\
\text { habitants ajustée sur les communes }\end{array}$ \\
\hline Autriche & $\begin{array}{l}\text { Zones de bâti continu de plus de } 2000 \\
\text { habitants et moins de } 15 \% \text { de population } \\
\text { agricole, ajustée sur les communes. }\end{array}$ \\
\hline
\end{tabular}


Au Luxembourg, les localités, échelon infracommunal, sont les unités constitutives de base des agglomérations urbaines; en dehors d'une continuité de construction ténue, une agglomération se définit en outre par un certain nombre de caractères socioéconomiques (densité, poids de l'agriculture...) et une population supérieure à 10000 habitants. Il en existe cinq dans tout le pays. Élargies à l'ensemble des communes qui les englobent et après regroupement, elles forment trois régions urbaines : les aires urbaines du centre (Luxembourg) et du sud (Esch-surAlzette) et la zone urbaine du nord (EttelbruckDiekirch).

En Angleterre et au Pays de Galles, l'Enumeration District, échelon statistique infracom- munal, est l'unité statistique de base à partir de laquelle sont délimitées les urban areas. En Écosse, le système est un peu différent : le maillage infracommunal à partir duquel sont finalement délimitées les localities est celui des local authorities. Ne sont considérées comme localités urbaines que celles qui rassemblent au moins 1000 habitants permanents.

Dans d'autres pays, la délimitation des unités urbaines est effectuée à partir des maillages municipaux.

En France l'ajustement est effectué par rapport aux limites communales. Les communes qui possèdent une agglomération de population d'au moins 2000 habitants sont appelées

Encadré 3

\section{LA SUISSE : UNE DÉFINITION ORIGINALE}

Frontalière de la France, mais n'appartenant pas à I'Union européenne, la Suisse compte deux agglomérations s'étendant en partie sur le territoire français, Bâle et Genève.

Voici plus d'un siècle que la Suisse définit ses agglomérations urbaines. Jusqu'en 1980, cette définition reposait essentiellement sur des critères morphologiques (continuité de la zone bâtie). Depuis cette date, une nouvelle définition des agglomérations a été retenue ; elle se distingue de celle de la plupart des pays de l'Union européenne, dans la mesure où elle n'est ni fondée sur le statut juridique des localités, ni centrée sur la notion de zone de bâti continu (encore qu'il soit fait référence à cette dernière), mais s'appuie sur des critères fonctionnels (nombre d'emplois, déplacement domicile-travail, densité...).

Elle est désormais la suivante.

a) Une agglomération est un ensemble d'au minimum 20000 habitants, formé par la réunion des territoires de communes adjacentes.

b) Toute agglomération possède une zone centrale qui est formée d'une commune-noyau et, suivant le cas, d'autres communes qui remplissent les conditions suivantes : elles doivent compter 2000 emplois ou plus, et le quotient du nombre des personnes travaillant sur leur territoire par celui des personnes actives occupées y résidant doit être supérieur ou égal à 0,85 . En outre, ces communes doivent soit former une zone bâtie continue avec la commune-noyau ou avoir une frontière commune avec elle, soit y envoyer travailler au minimum un sixième de leur population active occupée.

c) Une commune n'appartenant pas à ladite zone centrale sera rattachée à l'agglomération si au moins un sixième de sa population résidente active occupée travaille dans la zone centrale définie précédemment et si au moins trois des cinq conditions figurant ci-dessous sont remplies :

1 - Il doit exister un lien de continuité de la zone bâtie entre une telle commune et la commune-noyau de l'agglomération. En d'autres termes, il ne doit pas y avoir de zone non bâtie (terres agricoles ou forêts) dépassant deux cents mètres.

2 - La densité combinée habitants/emplois par hectare de surface d'habitat et d'agriculture (sans les alpages) doit être supérieure à 10 .

3 - La population doit s'être accrue de plus de dix points par rapport à la moyenne nationale au cours des dix dernières années (ce critère n'est valable que pour les communes qui ne font pas encore partie d'une agglomération ; pour les autres, il sera considéré comme acquis indépendamment du taux atteint).

4 - Au minimum un tiers de la population active occupée résidente doit travailler dans la zone centrale. Pour les communes jouxtant deux agglomérations, cette condition sera également remplie si $40 \%$ au moins de leur population active occupée résidente travaille dans les deux zones centrales, dont au minimum un sixième dans l'une ou l'autre.

5 - La proportion de personnes résidantes travaillant dans le secteur primaire ne doit pas dépasser le double de la moyenne nationale (pour 1990, en raison du recul massif de la moyenne nationale de $6,2 \%$ à $4,1 \%$ et en dérogation à cette disposition, la valeur limite a été fixée à $10,3 \%$, c'est-à-dire que seul le recul net de 2,1 puints a été pris en compte).

Les communes de plus de 10000 habitants sont appelées "villes"; elles forment avec les agglomérations un espace dénommé " zone urbaine". 
"villes isolées». Lorsque l'agglomération s'étend sur plusieurs communes, on délimite des agglomérations urbaines multicommunales en identifiant sur le territoire national toutes les zones bâties (agglomérations de population) qui représentaient au moins 2000 habitants. Parmi les communes ainsi réunies, toutes celles dont l'agglomération de population représente moins de la moitié de la population totale de la commune sont éliminées. Si les communes non éliminées sont au moins au nombre de deux, et si la partie de leur population, résidant dans la zone bâtie, atteint au total au moins 2000 habitants, ces communes constituent une « agglomération urbaine multicommunale».

En Autriche, les statisticiens définissent, pour les besoins d'information des organisations internationales, les unités urbaines comme les communes où se trouve une zone bâtie de plus de 2000 habitants, et où moins de $15 \%$ de la population vit de l'agriculture.

En Grèce, une agglomération urbaine est un groupe de municipalités ou de communes qui sont urbaines et voisines, c'est-à-dire que moins de 200 mètres séparent les maisons de leur localité qui comprend au moins 10000 habitants. Au recensement de 1971, 13 agglomérations urbaines multicommunales ont ainsi été définies, et redélimitées en 1981. La population urbaine est définie comme la population totale des municipalités ou des communes dans lesquelles le plus grand noyau de population a 10000 habitants ou plus, et inclut également la population de ces 13 agglomérations urbaines, quelle que soit la population des communes qui les composent.

En Allemagne, plusieurs approches sont retenues pour définir les agglomérations. Le

\section{Encadré 4}

\section{LA COMPARAISON INTERNATIONALE DES VILLES :}

UNE IDÉE CENTENAIRE

Il y a un siècle, P. Meuriot (1897) effectuait déjà une comparaison des périmètres des agglomérations pour les plus grandes villes européennes (Paris, Londres, Berlin, etc.). Quelques années plus tard (1911), il tentait de réunir un consensus international sur la définition de l'agglomération et sur les règies à suivre pour la déterminer. Les règles qu'il proposait étaient fondées sur la taille des communes, leur densité, la distance au centre ville et les mouvements des personnes qui travaillent dans la ville et habitent au dehors. Ces propositions ne furent finalement pas retenues.

En 1934, Henri Bunle, futur directeur de la Statistique générale de la France présentait à son tour, à la XXIIe session de l'Institut international de statistique, à Londres, une communication intitulée : "Comparaison internationale des agglomérations urbaines". II constatait alors que :

"L'utilité d'une règle uniforme pour la détermination de l'agglomération urbaine, dans le but de comparaisons internationales, devient plus impérieuse que jamais.

II importe donc d'examiner les moyens qui, jusqu'à présent, ont été reconnus comme susceptibles de conduire à cette détermination :

1. Densité de la population des circonscriptions adjacentes ;

2. Augmentation du nombre des habitants dans ces mêmes circonscriptions ; comparaison avec l'ac- croissement moyen de la population dans l'arrondissement dont elles font partie ;

3. Détermination des migrations alternantes entre ces mêmes localités et le centre urbain."

Malheureusement, cet examen des statistiques disponibles menait à une conclusion pessimiste, puisque, selon H. Bunle (1934), " il paraît presque impossible d'établir une définition précise de l'agglomération urbaine sur le plan international, étant donné la complexité de la question. (...) D'où l'absolue nécessité de simplifier le problème pour faciliter les comparaisons. Plus modestement, plus fructueusement aussi semble-t-il du point de vue international, on peut rechercher une solution approchée, qui ne donne pas toute lagglomération, mais en délimite la fraction la plus importante et la plus intéressante, quant au nombre des habitants tout au moins ".

Estimant que " la considération de la densité [était] celle qui [répondait] le mieux au but limité poursuivi ", il détermina le périmètre des agglomérations de Paris, Londres et Berlin, en ne retenant que les circonscriptions administratives où la densité des habitants dépassait 1000 au $\mathrm{km}^{2}$. Ainsi délimitée, l'agglomération de Londres comptait 7,9 millions d'habitants, celle de Paris 5,6 millions, et celle de Berlin 3,5 millions.

En conclusion de sa communication, $\mathrm{H}$. Bunle affirmait que ces territoires étaient " certainement mieux comparables (...) que Paris, Gross Berlin et Greater London ». 
Bundesforschungsanstalt für Landeskunde und Raumordnung (Institut fédéral de recherche pour l'aménagement du territoire) délimite entre autres officiellement des «Verdichtungs und Ordnungsräume ». En raison de la réforme territoriale dans les nouveaux Länder, une nouvelle définition est en train de se mettre en place.

Il n'y a pas de définition officielle des agglomérations au Luxembourg, aux Pays-Bas, en Italie, en Espagne, ni en Finlande. Dans certains de ces pays on distingue néanmoins les communes rurales des communes urbaines (voire semi-urbaines) en s'appuyant sur des critères socioéconomiques (Pays-Bas, Italie), sur la taille de la commune (Espagne) ou sur le nombre de personnes vivant dans le noyau aggloméré (Finlande). Ces définitions n'ont pas de caractère officiel et sont en général uniquement établies pour servir de base à des études de type statistique.

\section{Le cas français}

La comparaison de ces définitions de l'urbain montre que la France fait partie des pays où elle est la plus extensive : seuil retenu pour définir la continuité du bâti fixé à 200 mètres, prise en compte de la totalitć d'unc commune dans l'espace urbain si seulement la moitié de sa population fait partie d'une agglomération multicommunale et surtout, seuil de population fixé à 2000 habitants. Ce dernier chiffre est souvent critiqué. Pendant longtemps, il a correspondu à une réalité démographique : au-dessous de ce seuil, les communes perdaient de la population par le jeu des mouvements migratoires ; au-dessus elles en gagnaient. Mais depuis une trentaine d'années, tous ces points de repère ont été bouleversés par le mouvement de périurbanisation, alors que certaines parties du territoire se vidaient inexorablement. Le seuil de 2000 habitants correspond maintenant souvent à un gros bourg, chef-lieu de canton, ayant conservé un rôle d'animation de l'espace rural qui l'entoure ; les fonctions qu'il assure ne sont manifestement pas de type urbain, et il n'y a qu'en France qu' une telle commune est classée parmi les unités urbaines. Un éventuel changement de définition, s'il surve- nait, devrait cependant se faire avec prudence, tant sont nombreux les textes législatifs ou réglementaires faisant référence à cette définition de l'urbain et du rural.

\section{Une tentative d'harmonisation}

Le souci de comparaison pousse naturellement à harmoniser les définitions entre les pays (cf. encadré 4). La tentative la plus achevée, réalisée par le Nurec, avec l'aide d'Eurostat, a conduit à la publication d'un atlas des agglomérations dans l'Union européenne. Limité aux agglomérations de plus de 100000 habitants, il retient une définition proche du concept français ; cependant, pour qu'une unité locale (commune, district, $\mathrm{Ge}$ meinde, ...) soit intégrée à l'agglomération, il suffit qu'une partie de son territoire appartienne à l'agrégat bâti élémentaire, sans qu'il soit imposé que cette fraction de territoire abrite au moins la moitié de la population. Le résultat permet effectivement une amorce de comparaison entre les plus grandes villes d'Europe. Il révèle des réalités qu'un point de vue trop étroitement national avait occultées : ainsi Lille apparait comme l'extrémité occidentale d'une immense agglomération couvrant une grande partie de la Belgique (dont les villes d'Anvers, Gand, Bruxelles), débordant même légèrement sur les Pays-Bas et regroupant plus de sept millions d'habitants. L'intérêt de cet exercice est aussi de mettre en évidence le fait, déjà souligné, que derrière des définitions formellement identiques, apparaissent des divergences dues à un maillage de base très hétérogène : les agglomérations anglaises, fondées sur le district (en moyenne 120000 habitants), sont difficilement comparables avec les françaises dont les communes sont de taille nettement plus faible (en moyenne 1500 habitants). Le chemin qui mène à la comparabilité totale des agglomérations entre les pays est encore bien long... Cependant, le travail entrepris à l'échelle mondiale par MoriconiEbrard (1994) pour définir de manière comparable les quelque 26000 unités urbaines de plus de 10000 habitants, à partir de l'ensemble des sources disponibles, montre que ce projet n'est pas insurmontable. Sa base de données Geopolis représente à ce jour la meilleure source de comparaison existante, pour l'ensemble des villes du monde de 1950 à 1990. 


\section{BIBLIOGRAPHIE}

Brunet R. (sous la direction de) (1989), Les villes européennes, La documentation française.

Bunle H. (1934), Comparaison internationale des agglomérations urbaines, Institut international de statistique, $\mathrm{XXII}^{\mathrm{e}}$ session, Londres.

Cattan N., Pumain D., Rozenblat C. et Saint-Julien Th. (1994), Le système des villes européennes, Paris, Anthropos.

Insee (1990), « Composition communale des unités urbaines, population et délimitation ", Recensement général de la population de 1990.

Meuriot P. (1897), Des agglomérations urbaines dans l'Europe contemporaire. Essai sur les causes, les conditions et les conséquences de leur développement, Paris, Berlin.

Moriconi-Ebrard F. (1991), « Les 100 plus grandes villes du monde », Économie et Statistique, juillet-août, $\mathrm{n}^{\circ} 245$, pp. 7-18.
Moriconi-Ebrard F. (1994), Geopolis, pour comparer les villes du monde, Paris, Anthropos, coll. «Villes».

Nurec (1994), «Atlas of Agglomerations in the European Union ", Network on Urban Research in the European Union, (Formerly European Community), Duisburg, trois volumes.

OCDE (1988), Statistiques urbaines dans les pays de l'OCDE, Programme des affaires urbaines.

Pumain D., Saint-Julien Th., Cattan N. et Rozenblat C. (1992), Le concept statistique de la ville en Europe, Eurostat, Opoce, Ined, équipe « Paris »/CNRS, Nurec.

Schuler M. et Joye D. (avec la collaboration de) (1994), Les niveaux géographiques de la Suisse: recensement fédéral de la population 1990, Office fédéral de la Statistique. 